\title{
REVIEW
}

\section{Morphological and physiological interactions of NG2-glia with astrocytes and neurons}

\author{
Rebekah Wigley, ${ }^{1 *}$ Niki Hamilton, ${ }^{2 *}$ Akiko Nishiyama, ${ }^{3}$ Frank Kirchhoff ${ }^{4}$ and Arthur M. Butt ${ }^{1}$ \\ ${ }^{1}$ Institute for Biomedical and Biomolecular Sciences, School of Pharmacy and Biomedical Sciences, University of Portsmouth, UK \\ ${ }^{2}$ Department of Physiology, University College London, UK \\ ${ }^{3}$ Department of Physiology and Neurobiology, University of Connecticut, USA \\ ${ }^{4}$ Neurogenetics, Max Planck Institute of Experimental Medicine and ${ }^{5}$ DFG Research Center for Molecular Physiology of the Brain, \\ Goettingen, Germany
}

\begin{abstract}
Models of central nervous system (CNS) function have historically been based on neurons and their synaptic contacts - the neuronal doctrine. This doctrine envisages glia as passive supportive cells. However, electrophysiological and imaging studies in brain slices show us that astrocytes, the most numerous cells in the brain, express a wide range of neurotransmitter receptors that are activated in response to synaptic activity. Furthermore, astrocytes communicate via calcium signals that are propagated over long distances by the release of 'gliotransmitters', the most abundant being adenosine triphosphate (ATP). This has led to the concept of the neuron-astroglial functional unit as the substrate of integration in the CNS. Recently, a novel glial cell type has been characterized by expression of the proteoglycan NG2. These NG2-glia receive presynaptic input from neurons and responds to neurotransmitters released at synapses. Now, studies on transgenic mice in which fluorescent proteins are specifically expressed by subclasses of glia are helping to address the question of where NG2-glia fit in the neuron-astroglial model of integrated brain function. NG2-glia, as well as astrocytes, have been shown to respond to neuronal and astroglial signals by raised intracellular calcium, which is a potential communications mechanism by which NG2-glia may be active partners in neuron-glial circuits. Moreover, a current concept of NG2-glia considers them to be 'neural stem cells' and an exciting prospect is that neuron-glial signalling may regulate the differentiation capacity of NG2-glia and their response to injury.
\end{abstract}

Key words AMPA; astrocyte; ATP; axon; glutamate; neuron; NG2; NMDA; oligodendrocyte; precursor cell; purine; purinoreceptor.

\section{Introduction}

Glial cells express a range of neurotransmitter receptors that are coupled to elevations in intracellular $\left[\mathrm{Ca}^{2+}\right]$ (Verkhratsky \& Kettenmann, 1996; Deitmer et al. 1998; Alberdi et al. 2005; Butt 2006). Glutamate released at synapses evokes $\mathrm{Ca}^{2+}$ signals in neighbouring astroglia,

\section{Correspondence}

Professor Arthur M. Butt, School of Pharmacy and Biomedical Sciences, University of Portsmouth, St Michael's Building, White Swan Road, Portsmouth PO1 2DT, UK. E: arthur.butt@port.ac.uk

*N.H. and R.W. contributed equally to the writing of this paper.

Accepted for publication 24 March 2007 which activates various signalling pathways that can regulate their release of neurotransmitters (Araque et al. 1999; Haydon, 2001). Astroglia release the excitatory neurotransmitters glutamate and adenosine triphosphate (ATP) (Nedergaard, 1994; Parpura et al. 1994), and the latter is metabolized by ectonucleotidases to adenosine, which can be inhibitory for neurons (Newman, 2004; Pascual et al. 2005). A single astroglial cell can contact multiple synapses and so a $\mathrm{Ca}^{2+}$ signal evoked in response to glutamate released at one synapse can result in the release of transmitters from astroglia to exert excitatory or inhibitory effects on other synapses contacted by the same cell (Newman, 2004; Pascual et al. 2005). Moreover, $\mathrm{Ca}^{2+}$ signals are propagated 
over long distances through the astroglial syncytium, both through gap junctions and by the release of ATP, so that astroglia can influence neuronal activity at synapses distant to the initiating synaptic event (Dani et al. 1992; Cotrina et al. 2000; Newman, 2003). This is the functional substrate by which astroglia may serve as active partners to neurons in information processing and integration. Recent studies show that NG2-glia also contact synapses and nodes of Ranvier, and respond to neuronally released neurotransmitters (Butt et al. 1999; Bergles et al. 2000; Lin et al. 2004; Ziskin et al. 2007). NG2-glia are therefore a further element in neural circuits and this review will focus on their morphological and physiological interactions with astroglia and neurons.

\section{Relation of NG2-glia to oligodendrocyte and neural precursors}

NG2-expressing cells were first identified as oligodendrocyte progenitor cells (OPC) on the basis of their antigenic phenotype (Levine \& Stallcup, 1987) and are equivalent to oligodendrocyte-type- 2 astrocyte (O-2A) cells, which can differentiate into oligodendrocytes or type-2 astrocytes, depending on culture medium (Raff et al. 1983). In vivo studies on the rodent brain have provided evidence that NG2-expressing OPCs give rise to myelin-forming oligodendrocytes, but a substantial population of NG2-expressing glia persist in the adult central nervous system (CNS); these have been termed 'synantocyes' or 'polydendrocytes' (Butt et al. 2005; Nishiyama et al. 2005), but here we will use the more general term 'NG2-glia'. Adult NG2-glia in vivo are probably equivalent to the $\mathrm{O}-2 \mathrm{~A}$ cells isolated from adult CNS by ffrench-Constant \& Raff (1986), which can be driven in vitro to generate oligodendrocytes and astrocytes; Fulton et al. (1992) identified adult $\mathrm{O}-2 \mathrm{~A}$ cells in vivo in the optic nerve using cobalt uptake through AMPA-type glutamate receptors and these are morphologically identical to NG2-glia subsequently described in the optic nerve (Butt et al. 1999). Furthermore, studies in transgenic mice in which expression of green fluorescent protein (GFP) was driven by glial fibrillary acidic protein (GFAP) or 2', 3'-cyclic nucleotide $3^{\prime}$-phosphodiesterase (CNP) indicated that NG2-glia have some astroglial properties (Matthias et al. 2003; Grass et al. 2004) and may contribute to the genesis of neurons (Belachew et al. 2003; Aguirre \& Gallo, 2004; Aguirre et al. 2004). Hence, OPCs/NG2-glia have the capacity to be multipotent neural stem cells (Kondo \&
Raff, 2000; Belachew et al. 2003). A number of studies have provided substantial evidence that adult NG2-glia can regenerate oligodendrocytes in vivo (Polito \& Reynolds, 2005), but it is not yet certain that adult $\mathrm{NG2-glia}$ retain multipotent stem cell properties in vivo.

\section{NG2-glia and astroglia are separate populations with overlapping distributions}

We have examined the interrelationships between NG2-glia, astrocytes and neurons in transgenic mice. To identify NG2-glia and astrocytes unequivocally we took advantage of two different transgenic mouse lines. In one developed by Akiko Nishiyama, NG2-glia can be identified by expression of the red fluorescent protein DsRed (from the sea anemone Discosoma sp.), which is coexpressed with the NG2 chondroitin sulphate proteoglycan (CSPG) due to genomic, BAC-mediated NG2DsRed transgene insertion (Ziskin et al. 2007). In the other mouse line, astrocytes were identified by enhanced green fluorescent protein (EGFP) expressed under the control of the human GFAP promoter (Nolte et al. 2001). In NG2/DsRed mice, immunolabelling confirmed that the vast majority of DsRed+ cells are labelled by anti-NG2 antibodies (Fig. 1A,B), consistent with previous findings (Ziskin et al. 2007); the NG2 CSPG is also expressed by pericytes surrounding the brain microvasculature, and so both NG2-glia and blood vessels express DsRed. Notwithstanding this, the entire cellular morphology and process aborization of NG2-glia are clearly resolved by DsRed expression and by immunohistochemistry (Fig. 1A,B). In contrast, there is not perfect co-localization of GFAP immunolabelling and expression of EGFP; there are numerous GFAP+/EGFP- cells and EGFP expression appears greatest in protoplasmic astrocytes (Fig. 1C); due to the particular cell line, the transgenic expression labels astrocytes (GFAP+) but also some NG2-glia, thereby suggesting that NG2-glia and astrocytes have some common properties (Matthias et al. 2003; Grass et al. 2004). However, the beauty of EGFP is that it is distributed throughout the whole cell, contrasting dramatically with GFAP immunohistochemistry, which labels only the major processes of astrocytes and gives no idea of their true morphological complexity (Fig. 1D).

NG2-glia and astrocytes are both stellate cells and have similar distributions throughout the grey and white matter (Fig. 2). However, NG2-glia and astrocytes differ in the organization of their process arborizations and structure. NG2-glia emanate fine processes that 

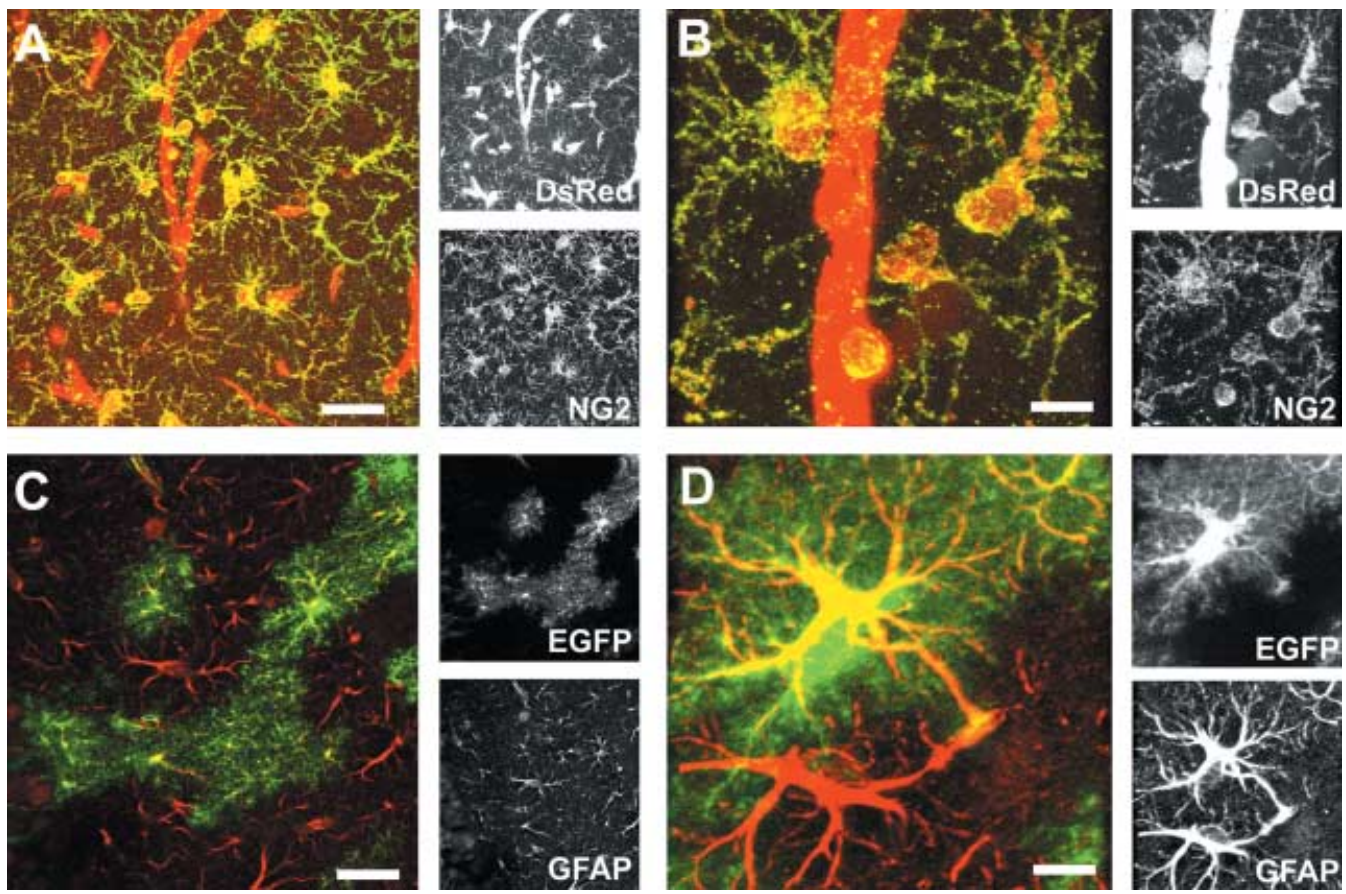

Fig. 1 NG2-glia and astroglia visualized in two different transgenic mouse lines. Following fixation in $4 \%$ paraformaldehyde, brains from NG2-DsRed mice $(A, B)$ and GFAP-EGFP mice $(C, D)$, aged postnatal day $(P) 15-30$, mid-sagital sections were cut at $50 \mu \mathrm{m}$ on a vibratome and immunolabelled for either NG2 (A,B) or GFAP (C,D), using immunohistochemistry protocols described previously (Butt et al. 1999). Images were captured on a Zeiss 510 meta confocal microscope at $543 \mathrm{~nm}$ (red) and $488 \mathrm{~nm}$ (green). $(A, B)$ Cerebral cortex showing NG2-DsRed fluorescence and immunolabelling with anti-NG2 antibodies (green). The vast majority of NG2-glia coexpress DsRed and NG2 (coexpression appears yellow). (C,D) Cerebral cortex showing GFAP-EGFP fluorescence (green) and immunolabelling with anti-GFAP antibodies (red). In this particular cell line, EGFP expression is not observed in all GFAP+ astrocytes. GFAP immunohistochemistry labels the major processes of astrocytes, whereas EGFP is distributed throughout the entire process arborization. Scale bars $=25 \mu \mathrm{m}$ in $A, C$ and $10 \mu \mathrm{m}$ in B,D.

first branch close to the cell body $(\leq 10 \mu \mathrm{m})$, and appear to bifurcate repeatedly and taper to form $\geq 50$ termini within approximately $50 \mu \mathrm{m}$ of the cell body (Fig. 2A). Astrocytes have four or more dense primary processes, which branch in an alternate fashion, and bear numerous fine collateral processes (Fig. 2B); the primary processes contain the GFAP intermediate filament and are therefore GFAP-immunopositive, whereas the fine processes cannot be visualized by GFAP immunolabelling (Fig. 1D). NG2-immunopositivity has been reported in dimly EGFP-expressing cells in the hippocampus and brain stem (Matthias et al. 2003; Grass et al. 2004). We did not observe NG2 immunolabelling in brightly EGFP+ astrocytes (Fig. 2C,D), nor did we observe GFAP immunopositivity in DsRed+ NG2-glia (Fig. 2E,F).

\section{Anatomical and physiological interactions between NG2-glia, astroglia and neurons}

The anatomical relations between NG2-glia, astroglia and neurons are illustrated in the cerebellum (Figs 3 and 4). Cerebellar EGFP+ astroglia comprise astrocytes within the granular cell layer and white matter, and Bergmann glia, which have cell bodies located within the Purkinje cell layer and primary processes that extend to the pial surface parallel to the dendritic trees of the Purkinje cells (Fig. 3A-C). Innumerable short, fine collaterals extend from the primary processes of Bergmann glia and these encapsulate synapses within the Purkinje cell dendritic tree (Grosche et al. 1999, 2002). NG2-glia are located throughout the white matter, granular cell layer and molecular layers (Fig. 3D). NG2-glial cell processes extend radially through the molecular cell layer to form multiple associations with Bergmann glia (Fig. 3E) and with Purkinje cells (Fig. 3F,G). Ultrastructural and electrophysiological studies have shown that these NG2-glial cell processes contact synapses between climbing fibres and Purkinje cells (Lin et al. 2004). Equivalent synaptic relations have been demonstrated in the hippocampus (Bergles et al. 2000), and it is conceivable they exist throughout the CNS. Excitatory synapses are formed by parallel and climbing fibres 

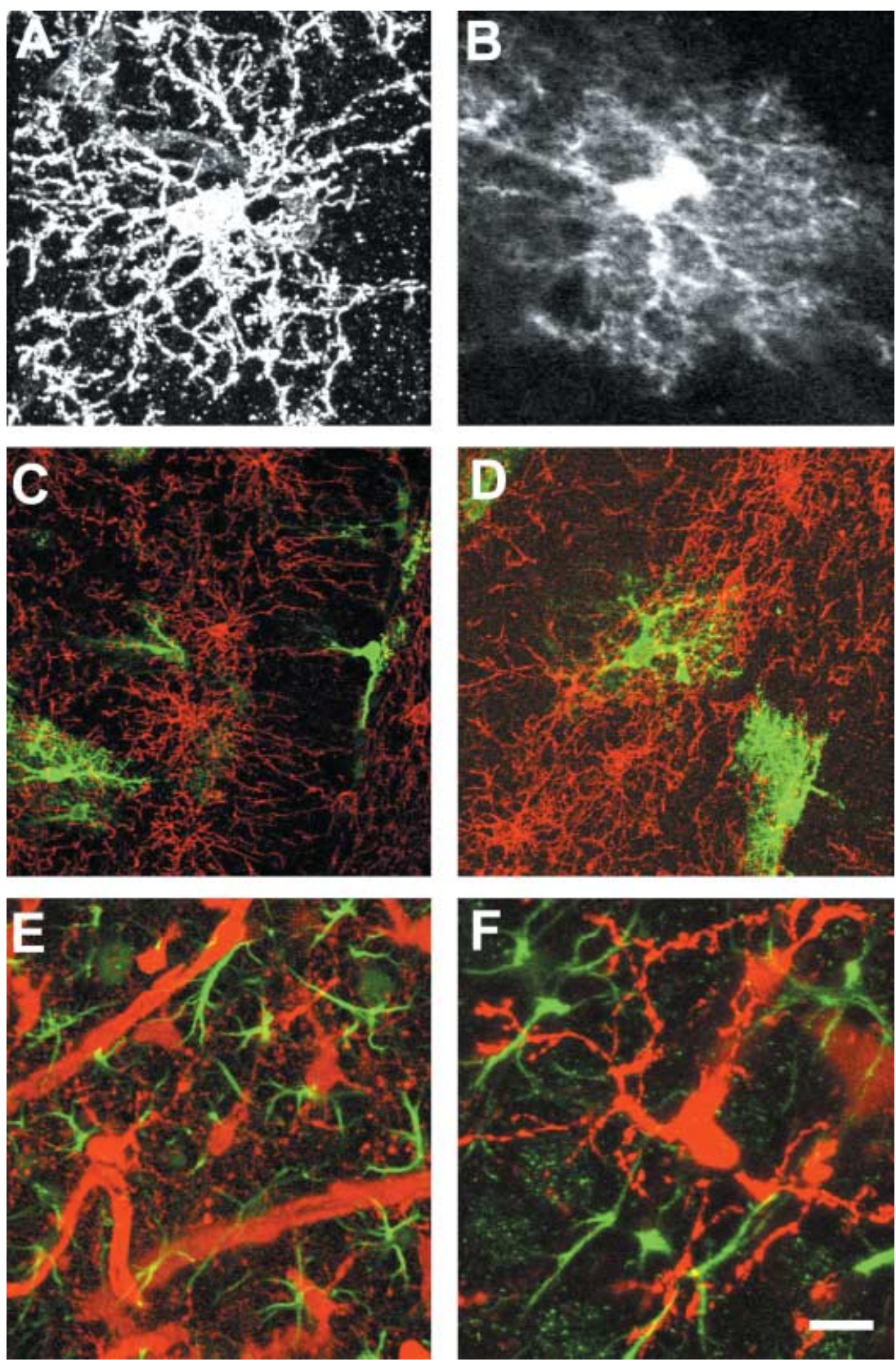

Fig. 2 Morphology and distribution of NG2-glia and astrocytes. Confocal microscopic images of mid-sagittal sections of P15 brains from NG2-DsRed mice $(A, E, F)$ and GFAP-EGFP mice $(B-D)$, immunolabelled for NG2 $(C, D)$ or GFAP $(E, F)$. (A,B) NG2-glia and protoplasmic astrocytes are superficially similar, but differ in the organization of their process arborizations and structure. $(C, D)$ Immunolabelled NG2-glia (red) are a separate and distinct population from EGFP+ protoplasmic astrocytes (green). The process domains of NG2-glia and protoplasmic astrocytes overlap with each other. $(E, F)$ GFAP immunolabelling was not observed in DsRed+ NG2 glia; note the DsRed+ blood vessels in $E$. Scale bar $=25 \mu \mathrm{m}$ in $C, D$ and $10 \mu \mathrm{m}$ in $A, B, E, F$. impinging on the dendritic tree of Purkinje cells in the molecular layer of the cerebellum. Bergmann glia and NG2-glia contact or even intimately enwrap these synapses and respond to synaptic activity (Grosche et al. 1999; Lin \& Bergles, 2004), and are therefore functional elements in the cerebellar network. However, Bergmann glia and NG2-glia differ dramatically in their relations with neurons. A single Purkinje cell is served by multiple Bergmann glia (Fig. 4A), which extend processes in parallel to the Purkinje cell dendritic tree, and each Bergmann glial cell contains many hundreds of independent microdomains which are capable of autonomous interactions with the synapses they ensheath (Grosche et al. 1999, 2002). In contrast, NG2-glia have radially orientated processes, with domains that encompass multiple Bergmann glia and Purkinje cells (Fig. 4B). NG2-glia receive inputs from multiple climbing fibres (Lin et al. 2004). These interrelations between NG2-glia, astroglia and neurons in the cerebellum are illustrated in Fig. 5. In the adult mouse cerebellum, the width of the entire molecular layer is served by one or two NG2-glial cells, which cover non-overlapping domains 
Fig. 3 NG2-glia and Bergmann glia have different domains in the cerebellum. Confocal microscopic images of mid-sagittal sections of $P 19$ cerebellum from GFAP-EGFP mice $(A, B, C, E)$ and NG2-DsRed mice $(D, F, G)$, immunolabelled for calbindin $(A, B, C, F, G)$ or NG2 (E). (A-C) EGFP+ Bergmann glia (green) have cell bodies adjacent to calbindin-immunopositive Purkinje cells (red). A single Purkinje cell may be served by multiple Bergmann glia, which extend three or more primary processes from which pass innumerable fine collaterals that contact the dendritic trees of the Purkinje cells. (D) DsRed+ NG2-glia are located throughout the cerebellar layers (some Purkinje cells identified by asterisk), with processes that extend radially through the molecular layer $(\mathrm{ML})$, Purkinje cell layer $(\mathrm{PCL})$ and granule cell layer (GCL). (E) Immunopositive NG2-glial cell in the molecular cell layer extends processes that intertwine with the rising processes of multiple Bergmann glia. $(F, G)$ DsRed+ NG2-glial cells amongst the cell body (F) and dendritic trees (G) of calbindin-immunopositive Purkinje cells. Scale bar $=50 \mu \mathrm{m}$ in $A, 30 \mu \mathrm{m}$ in B-D, and $20 \mu \mathrm{m}$ in $\mathrm{E}-\mathrm{G}$.
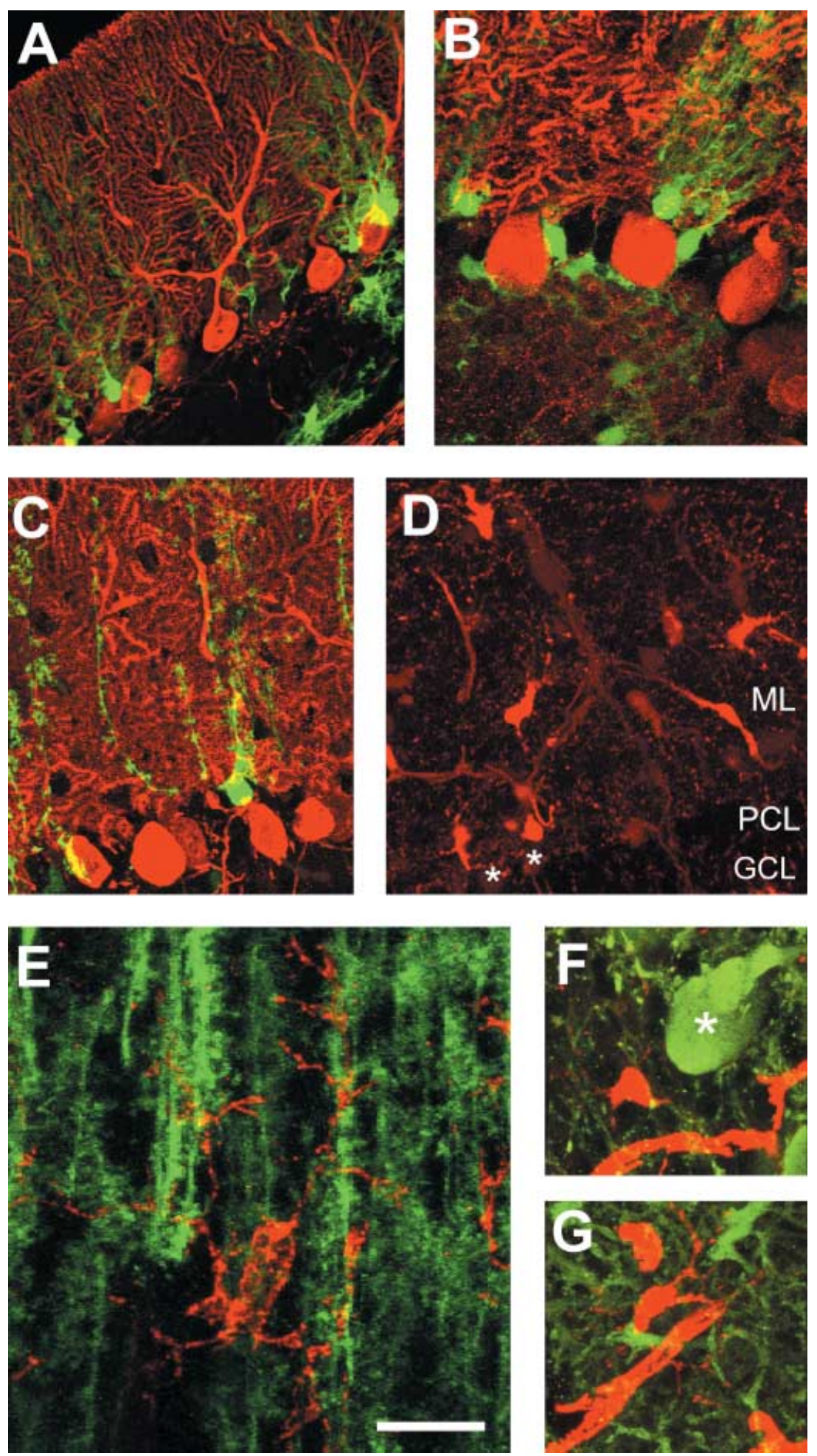

up to $100 \mu \mathrm{m}$ in diameter. It seems unimaginable that a single NG2-glial cell contacts all of the synapses within its domain (many millions), but this awaits a systematic study; cortical protoplasmic astrocytes cover the synapses of approximately 90000 synapses within their domain (Oberheim et al. 2006), and they have a far more complex process geometry than NG2-glia (Fig. 2A,B). NG2-glia have fairly uniform morphology and distribution throughout the brain, suggesting NG2-glia may serve the same functions in the different 

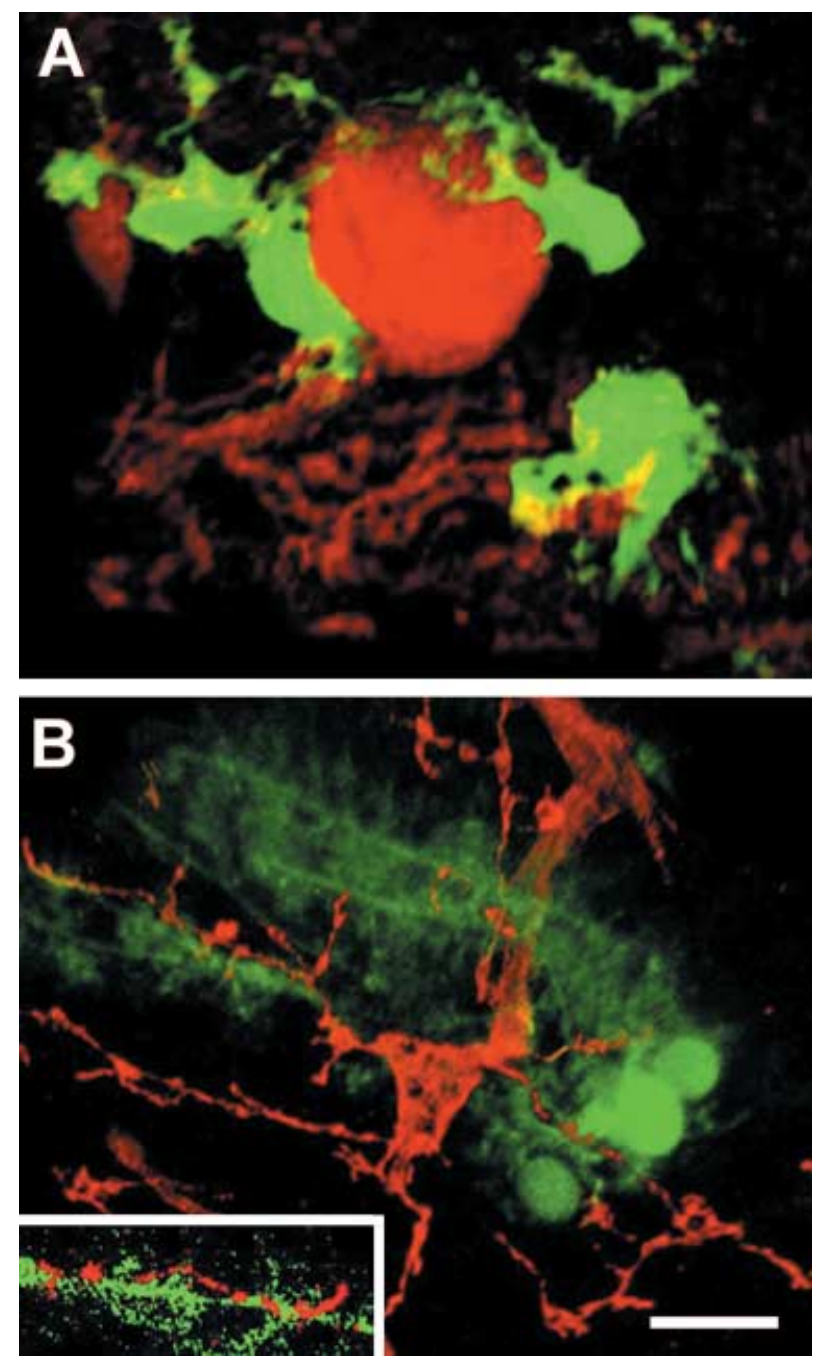

Fig. 4 Relations between Bergmann glia, Purkinje neurons and NG2-glia. Confocal microscopic images of mid-sagittal sections of P19 cerebellum from GFAP-EGFP mice, immunolabelled for calbindin (A) or NG2 (B), and deconvolved and rotated using Volocity (version 4.0.1; Improvision, UK). (A) Multiple Bergmann glia serve a single Purkinje cell, and each Bergmann glial cell contains many hundreds of independent microdomains which ensheath synapses. (B) NG2-glia process domains encompasses multiple Bergmann glia and form intricate associations with the processes of Bergmann glia that ensheath synapses (inset). NG2-glia receive inputs via these processes from multiple climbing fibres (Lin et al. 2004). Scale bar $=15 \mu \mathrm{m}$.

brain regions. For example, in the hippocamus, NG2-glia have a similar morphology and density as in cerebellum (Butt et al. 2005), and receive glutamatergic synaptic input from neurons (Bergles et al. 2000). In the hippocampus and cerebral cortex, both astrocytes (of the protoplasmic type) and NG2-glia are organized in radial domains (see Figs 1 and 2), but whereas the domains of the same cell type overlap very little, those between astrocytes and NG2-glia overlap completely (e.g. Fig. 2D). Domain organization in astrocytes is considered to be important for integrated activity at the synapses within their domain (Oberheim et al. 2006) and it may serve the same purpose in NG2-glia. In addition, NG2-glial cell domains may also serve to interconnect astroglial domains, or vice versa, as astrocytes communicate with NG2-glia (see below).

The numerous differences between NG2-glia and astroglia and their relationships with neurons indicate they may form distinct communications pathways with different functions within neural networks. A major function of astroglial cells is glutamate uptake at synapses. In Bergmann glia, glutamate uptake has been shown to play an essential role in the maintenance of the functional one-to-one relationship between climbing fibres and Purkinje cells, by preventing spill-over of glutamate (Takayasu et al. 2006). NG2-glia do not express glutamate transporters (Matthias et al. 2003; Zhou et al. 2006) and so do not perform this function at synapses. In contrast, both astroglia and NG2-glia have AMPA-type glutamate receptors which are activated by neural activity and result in raised intracellular calcium ([Ca $\left.\left.{ }^{2+}\right]_{i}\right)$ (Fig. 6; Grosche et al. 1999; Bergles et al. 2000; Lin et al. 2004). This activity in Bergmann glia regulates their structural and functional relations at glutamatergic synapses and, significantly, is essential for the innervation of Purkinje cells by climbing fibres (lino et al. 2001). An equivalent affect of NG2-glial cell activity on neurons has not been demonstrated, but in hippocampal NG2-glia, activation of $\mathrm{Ca}^{2+}$-permeable AMPA receptors mediates a form of activity-dependent 'long-term potentiation' (LTP), which is the hallmark of synaptic plasticity in neurons and underlies memory (Ge et al. 2006). Thus, NG2-glia are anatomically and physiologically integrated into neural networks, but it remains to be seen if they do more than follow neuronal activity.

\section{Glutamate and ATP in astroglial and NG2-glial cell physiology and pathology}

Astroglia communicate by $\mathrm{Ca}^{2+}$ signals propagated by the release of the 'gliotransmitter' ATP, which is the functional substrate of integrated neuron-astroglial units. We have therefore examined whether NG2-glia also display $\mathrm{Ca}^{2+}$ signalling in the optic nerve (Fig. 6; Hamilton, 2006). Astrocytes and NG2-glia both contact 
Fig. 5 Bergmann glia and NG2-glia are functional elements in the cerebellar network. Bergmann glia (green) and NG2-glia (black), respectively, ensheath and contact synapses formed by parallel fibres (light blue) and climbing fibres (red) on the dendritic tree of Purkinje cells (dark blue) in the molecular layer of the cerebellum. Bergmann glia are orientated parallel to the Purkinje cell dendritic tree, whereas NG2-glia are radially orientated, contacting multiple Purkinje cells and Bergmann glia. Bergmann glia and NG2-glia are anatomically and physiologically integrated into neural networks.

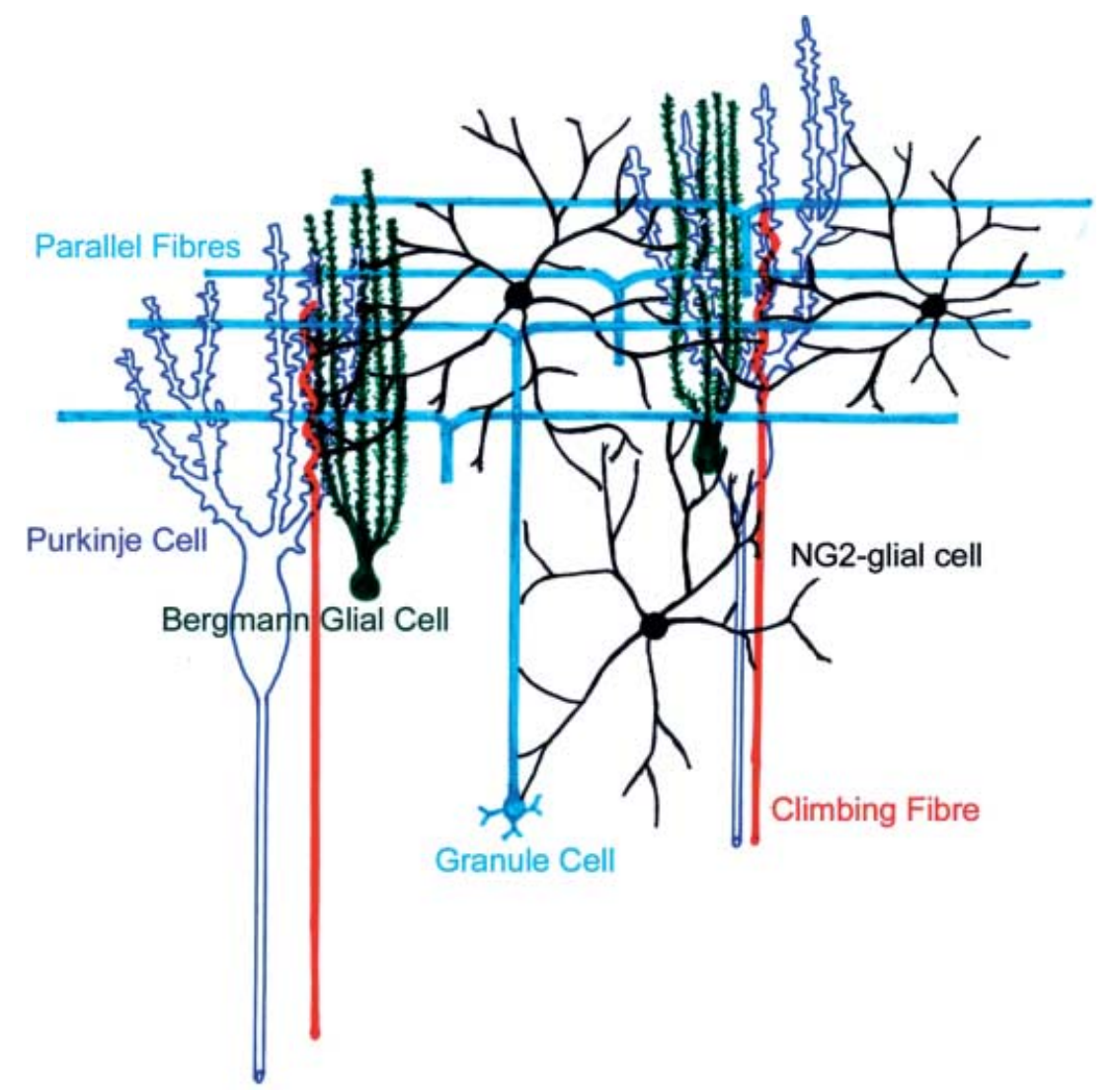

nodes of Ranvier, the sites of action potential propagation and only areas of axolemma not covered by myelin in the optic nerve, and therefore the site for direct signalling from axons to astrocytes and NG2-glia (Butt et al. 1994, 1999). In addition, vesicular release of glutamate from unmyelinated axons has been shown to evoke AMPA receptor-mediated currents in NG2-glia (Ziskin et al. 2007). In myelinated axons, each node of Ranvier is contacted by the perinodal microprocesses of astrocytes and NG2-glia, and individual astrocytes and NG2-glia contact multiple nodes of Ranvier (Butt et al. 1994, 1999, 2005). Astrocytes and NG2-glia in the mouse optic nerve display spontaneous $\mathrm{Ca}^{2+}$ signals (Fig. 6A), and elevations in $\left[\mathrm{Ca}^{2+}\right]_{i}$ are evoked in response to electrical stimulation of axons (Fig. 6B). Calcium signals in astrocytes and NG2-glia are mediated by glutamate and ATP (Fig. 6C); glutamate acts on both AMPA- and NMDA-type glutamate receptors in OPCs/NG2-glia and in astrocytes (Karadottir et al. 2005; Hamilton, 2006;

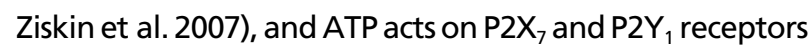
(James \& Butt, 2002; Hamilton, 2006). In addition, we have shown that ATP acts as a 'gliotransmitter' in the optic nerve propagating $\mathrm{Ca}^{2+}$ waves from astrocytes and to neighbouring NG2-glia (Fig. 6D). In these experiments, mechanical stimulation of astrocytes with a sharp microelectrode elicited a wave of $\mathrm{Ca}^{2+}$ that passed from cell to cell, including DsRed+ NG2-glia and EGFP+ astrocytes located within $100 \mu \mathrm{m}$ of the stimulus source, and which were markedly (and reversibly) inhibited by suramin (Fig. 6D). These studies on transgenic mice show for the first time that neuronal activity triggers $\mathrm{Ca}^{2+}$ signals in NG2-glia in situ, and provide evidence of ATP (gliotransmitter)-mediated communication between astrocytes and NG2-glia.

Glutamate- and ATP-evoked $\mathrm{Ca}^{2+}$ signalling provide mechanisms by which the overlapping domains of neurons, astroglia and NG2-glia are functionally connected. In astrocytes, this couples astroglial functions with neuronal activity, for example stimulating $\mathrm{K}^{+}$ uptake, glutamate uptake and glycogenolysis, and modulating neuronal activity (Verkhratsky \& Kettenmann, 1996; Deitmer et al. 1998). NG2-glia are not known to perform these astroglial functions. However, several studies provide evidence of roles for ATP and glutamate in the regulation of OPC differentiation and, by extension, they may regulate the neuro/gliogenic potential of 


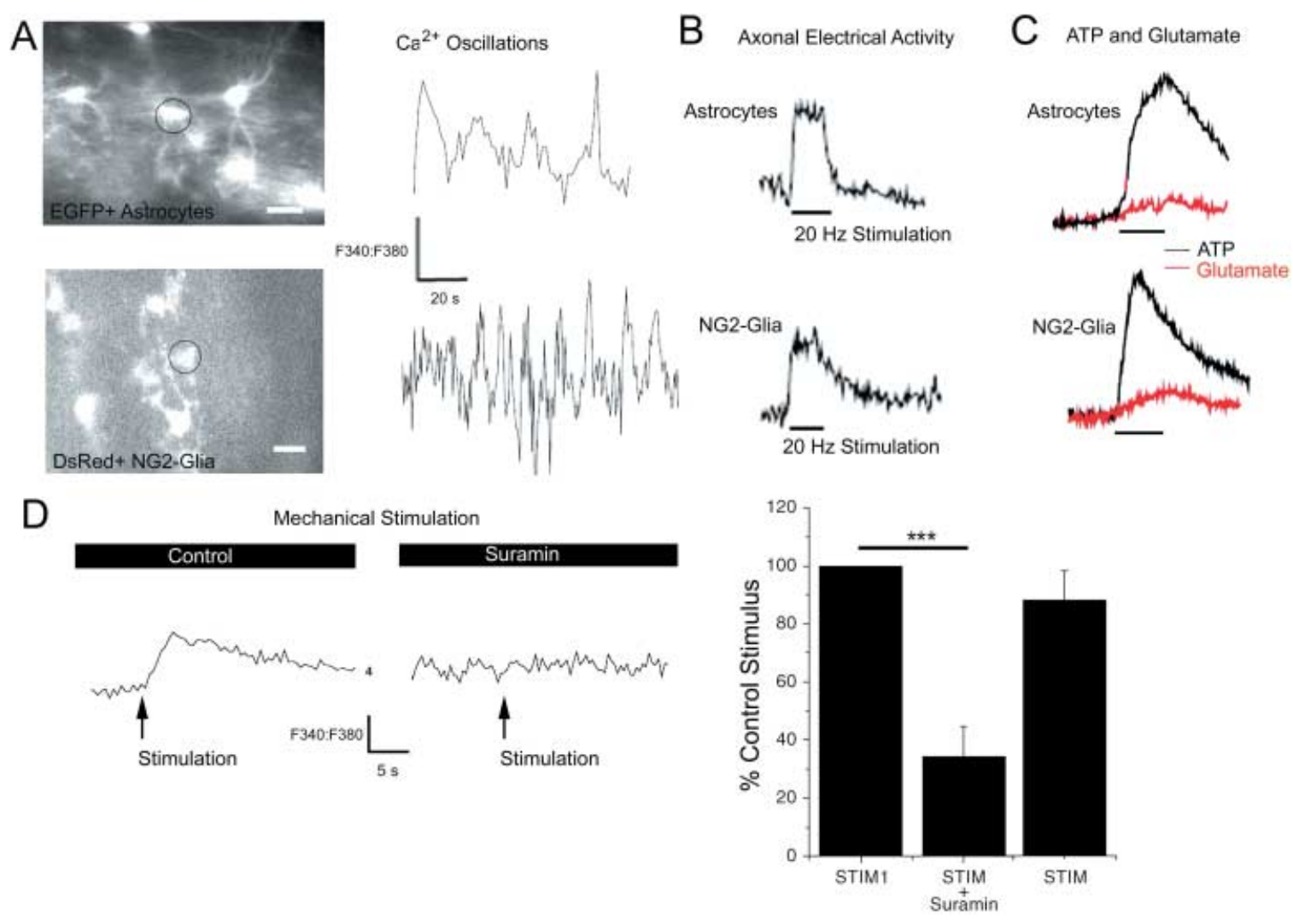

Fig. 6 Stimulation of axons in the optic nerve evokes calcium signalling in astrocytes and NG2-glia. Optic nerves from GFAP-EGFP mice and NG2-DsRed mice aged P15 were isolated intact and loaded with the calcium-sensitive dye fura-2, as described previously (James \& Butt, 2002). Cells were imaged on an Olympus upright microscope (BX50W1), and visualized using an Achroplan $\times 20$ water immersion lens and excited alternately at 340 and $380 \mathrm{~nm}$, using a Cairn monochromator (Cairn Research Ltd, UK). Emissions were detected at $510 \mathrm{~nm}$ using a Photometrics S-Coolsnap CCD camera (supplied by Cairn Research Ltd, UK). The monochromator and the CCD camera were controlled and synchronized by Axon Imaging Workbench 5.1 (Axon Instruments), and quantitative measurements were made using the same program. (A) Measurements were made from EGFP+ astrocytes and DsRed+ NG2-glia, and calcium variations expressed as the change in ratio after background subtraction (F340:F380). Spontaneous $\left[\mathrm{Ca}^{2+}\right]_{\mathrm{i}}$ oscillations were observed in both EGFP+ astrocytes and DsRed+ NG2-glia. (B) Electrical stimulation of the optic nerve to evoke axonal action potentials resulted in a rapid increase in $\left[\mathrm{Ca}^{2+}\right]_{i}$ in EGFP+ astrocytes and DsRed+ NG2-glia, which was sustained for the duration of the stimulus. (C) Bath administration of $100 \mu \mathrm{M}$ ATP or glutamate evoked raised [Ca $\left.{ }^{2+}\right]_{i}$ in EGFP+ astrocytes and DsRed+NG2-glia. (D) Mechanical stimulation of the optic nerve with a sharp microelectrode triggered raised glial [Ca $\left.{ }^{2+}\right]_{i}$ that was inhibited by the purinoreceptor antagonist suramin, indicating that ATP released by astrocytes propagates a calcium wave that passes to NG2-glia.

NG2-glia, in their capacity as neural stem cells (see above). Adenosine and ATP released during action potential propagation regulate differentiation of OPCs into oligodendrocytes, adenosine stimulating differentiation directly, whereas ATP acts indirectly by stimulating astrocytes to release leukaemia inhibitory factor (LIF) (Stevens et al. 2002; Ishibashi et al. 2006). Conversely, activation of AMPA receptors inhibits OPC proliferation and lineage progression (Borges et al. 1994; Gallo et al. 1996; Yuan et al. 1998). Thus, glutamate released at synapses may serve to maintain NG2-glia in a quiescent state, whereas ATP could stimulate proliferation and differentiation.
Over-activation of AMPA (and NMDA) receptors and $\mathrm{P} 2 \mathrm{X}_{7}$ purinoreceptors by high levels of glutamate and ATP, such as occur in pathology, trigger sustained $\mathrm{Ca}^{2+}$ influx in OPCs and astrocytes (James \& Butt, 2002; Alberdi et al. 2005). This may trigger cell death (Alberdi et al. 2005), but there is evidence in astrocytes that activation of purinoceptors may also stimulate reactive gliosis (Franke et al. 2001). Notably, NG2-glia undergo cell proliferation and hyperplasia following neuronal damage in the CNS (Tan et al. 2005), and it is possible that neurotransmitter-mediated $\mathrm{Ca}^{2+}$ influx may be involved in the injury response of NG2-glia. 


\section{Conclusions}

Neurons and glia are functionally interdependent and their physiology and pathology are integrated by extracellular signals, including glutamate and ATP. Neuronal activity triggers $\mathrm{Ca}^{2+}$ signalling in astrocytes and NG2-glia. In addition, astrocytes communicate with each other and with NG2-glia by $\mathrm{Ca}^{2+}$ waves propagated through astroglial release of ATP, which in turn can influence neuronal activity. This integrates astroglial and neuronal activity, and it may do the same in NG2-glia. However, astrocytes and NG2-glia do not serve the same functions. Primary functions of astrocytes are to provide physical support, and regulate extracellular potassium and uptake of glutamate at synapses. The only known functions of NG2-glia, by contrast, are to form the glial scar in response to CNS injury and to regenerate oligodendrocytes. Glutamateand ATP-mediated signalling provide potential mechanisms for regulating these functions of NG2-glia. It remains to be seen whether NG2-glia are also important in integrative neuronal activity.

\section{Acknowledgements}

This study was supported by the BBSRC. N.H. was in receipt of a PhD studentship from the Anatomical Society of Great Britain and Ireland. R.W. is in receipt of a Strategic PhD Studentship from the BBSRC.

\section{References}

Aguirre A, Chittajallu R, Belachew S, Gallo V (2004) NG2expressing cells in the subventricular zone are type C-like cells that contribute to interneuron generation in the postnatal hippocampus. J Cell Biol 165, 575-589.

Aguirre A, Gallo V (2004) Postnatal neurogenesis and gliogenesis in the olfactory bulb from NG2-expressing progenitors of the subventricular zone. J Neurosci 24, 10530-10541.

Alberdi E, Sanchez-Gomez MV, Matute C (2005) Calcium and glial cell death. Cell Calcium 38, 417-425.

Araque A, Parpura V, Sanzgiri RP, Haydon PG (1999) Tripartite synapses: glia, the unacknowledged partner. Trends Neurosci 22, 208-215.

Belachew S, Chittajallu R, Aguirre AA, et al. (2003) Postnatal NG2 proteoglycan-expressing progenitor cells are intrinsically multipotent and generate functional neurons. J Cell Biol 161, 169-186.

Bergles DE, Roberts JDB, Somogyi P, Jahr CE (2000) Glutamatergic synapses on oligodendrocyte precursor cells in the hippocampus. Nature 405, 187-191.

Borges K, Ohlemeyer C, Trotter J, Kettenmann H (1994)
AMPA/kainate receptor activation in murine oligodendrocyte precursor cells leads to activation of a cation conductance, calcium influx and blockade of delayed rectifying $\mathrm{K}^{+}$channels. Neuroscience 63, 135-149.

Butt AM, Duncan A, Berry M (1994) Astrocyte associations with nodes of Ranvier: ultrastructural analysis of HRP-filled astrocytes in the mouse optic nerve. J Neurocytol 23, 486-499.

Butt AM, Duncan A, Hornby MF, et al. (1999) Cells expressing the NG2 antigen contact nodes of Ranvier in adult CNS white matter. Glia 26, 84-91.

Butt AM, Hamilton N, Hubbard P, Pugh M, Ibrahim M (2005) Synantocytes: the fifth element. J Anat 207, 695-706.

Butt AM (2006) Neurotransmitter-mediated calcium signalling in oligodendrocyte physiology and pathology. Glia 54, 666675.

ffrench-Constant C, Raff MC (1986) Proliferating bipotential glial progenitor cells in adult rat optic nerve. Nature $\mathbf{3 1 9}$ 499-502.

Cotrina ML, Lin JH, Lopez-Garcia JC, Naus CC, Nedergaard M (2000) ATP-mediated glia signaling. J Neurosci 20, 28352844.

Dani JW, Chernjavsky A, Smith SJ (1992) Neuronal activity triggers calcium waves in hippocampal astrocyte networks. Neuron 8, 429-440.

Deitmer JW, Verkhratsky AJ, Lohr C (1998) Calcium signaling in glial cells. Cell Calcium 24, 405-416.

Franke H, Krugel U, Schmidt R, Grosche J, Reichenbach A, Illes $P$ (2001) P2 receptor-types involved in astrogliosis in vivo. $B r$ J Pharmacol 134, 1180-1189.

Fulton BP, Burne JF, Raff MC (1992) Visualization of O-2A progenitor cells in developing and adult rat optic nerve by quisqualate-stimulated cobalt uptake. J Neurosci 12, 48164833.

Gallo V, Zhou JM, McBain CJ, Wright P, Knutson PL, Armstrong RC (1996) Oligodendrocyte progenitor cell proliferation and lineage progression are regulated by glutamate receptormediated $\mathrm{K}^{+}$channel block. J Neurosci 16, 2659-2670.

Ge WP, Yang XJ, Zhang Z, et al. (2006) Long-term potentiation of neuron-glia synapses mediated by $\mathrm{Ca}^{2+}$-permeable AMPA receptors. Science 312, 1533-1537.

Grass D, Pawlowski PG, Hirrlinger J, et al. (2004) Diversity of functional astroglial properties in the respiratory network. J Neurosci 24, 1358-1365.

Grosche J, Matyash V, Moller T, Verkhratsky A, Reichenbach A, Kettenmann H (1999) Microdomains for neuron-glia interaction: parallel fiber signaling to Bergmann glial cells. Nat Neurosci 2, 139-143.

Grosche J, Kettenmann H, Reichenbach A (2002) Bergmann glial cells form distinct morphological structures to interact with cerebellar neurons. J Neurosci Res 68, 138-149.

Hamilton N (2006) A physiological study of NG2-glia and comparison with astrocytes. PhD thesis, London University.

Haydon P (2001) Glia. Listening and talking to the synapse. Nat Rev Neurosci 2, 185-193.

lino M, Goto K, Kakegawa W, et al. (2001) Glia-synapse interaction through $\mathrm{Ca}^{2+}$-permeable AMPA receptors in Bergmann glia. Science 292, 926-929.

Ishibashi T, Dakin KA, Stevens B, et al. (2006) Astrocytes promote myelination in response to electrical impulses. Neuron 49, 823-832. 
James G, Butt AM (2002) P2Y and P2X purinoceptor mediated $\mathrm{Ca}^{2+}$ signalling in glial cell pathology in the central nervous system. Eur J Pharmacol 447, 247-260.

Karadottir R, Cavelier P, Bergersen LH, Attwell D (2005) NMDA receptors are expressed in oligodendrocytes and activated in ischaemia. Nature 438, 1162-1166.

Kondo T, Raff M (2000) Oligodendrocyte precursor cells reprogrammed to become multipotential CNS stem cells. Science 289, 1754-1757.

Levine JM, Stallcup WB (1987) Plasticity of developing cerebellar cells in vitro studied with antibodies against the NG2 antigen. J Neurosci 7, 2721-2731.

Lin S-C, Huck JHJ, Roberts JD, Macklin WB, Somogyi P, Bergles DE (2004) Climbing fiber innervation of NG2-expressing glia in the mammalian cerebellum. Neuron 46, 773-785.

Matthias K, Kirchhoff F, Seifert G, et al. (2003) Segregated expression of AMP-type glutamate receptors and glutamate transporters defines distinct astrocyte populations in the mouse hippocampus. J Neurosci 23, 1750-1758.

Nedergaard M (1994) Direct signaling from astrocytes to neurons in cultures of mammalian brain cells. Science 263, 1768-1771.

Newman EA (2003) Glial cell inhibition of neurons by release of ATP. J Neurosci 23, 1659-1666.

Newman EA (2004) Glial modulation of synaptic transmission in the retina. Glia 47, 268-274.

Nishiyama A, Yang Z, Butt A (2005) Astrocytes and NG2-glia: what's in a name? J Anat 207, 687-693.

Nolte C, Matyash M, Pivneva T, et al. (2001) GFAP promotercontrolled EGFP-expressing transgenic mice: a tool to visualize astrocytes and astrogliosis in living brain tissue. Glia 33, 72-86.

Oberheim NA, Wang X, Goldman S, Nedergaard M (2006) Astrocytic complexity distinguishes the human brain. Trends Neurosci 29, 547-553.
Parpura V, Basarsky TA, Liu F, et al. (1994) Glutamate-mediated astrocyte-neuron signalling. Nature 369, 744-747.

Pascual O, Casper KB, Kubera C, et al. (2005) Astrocytic purinergic signaling coordinates synaptic networks. Science 310, 113-116.

Polito A, Reynolds R (2005) NG2-expressing cells as oligodendrocyte progenitors in the normal and demyelinated adult central nervous system. J Anat 207, 707-716.

Raff MC, Miller RH, Noble M (1983) A glial progenitor cell that develops in vitro into an astrocyte or an oligodendrocyte depending on culture medium. Nature 303, 390-396.

Stevens B, Porta S, Haak LL, Gallo V, Fields RD (2002) Adenosine: a neuron-glial transmitter promoting myelination in the CNS in response to action potentials. Neuron 36, 855868.

Takayasu Y, lino M, Shimamoto K, Tanaka K, Ozawa S (2006) Glial glutamate transporters maintain one-to-one relationship at the climbing fiber-Purkinje cell synapse by preventing glutamate spillover. J Neurosci 26, 6563-6572.

Tan AM, Zhang W, Levine JM (2005) NG2: a component of the glial scar that inhibits axon growth. J Anat 207, 717725.

Verkhratsky A, Kettenmann H (1996) Calcium signaling in glial cells. Trends Neurosci 19, 346-352.

Yuan X, Eisen AM, McBain CJ, Gallo V (1998) A role for glutamate and its receptors in the regulation of oligodendrocyte development in cerebellar tissue slices. Development 125, 2901-2914.

Zhou M, Schools GP, Kimelberg HK (2006) Development of GLAST(+) astrocytes and NG2(+) glia in rat hippocampus CA1: mature astrocytes are electrophysiologically passive. J Neurophysio/ 95, 134-143.

Ziskin JL, Nishiyama A, Rubio M, Fukaya M, Bergles DE (2007) Vesicular release of glutamate from unmyelinated axons in white matter. Nature Neurosci 10, 321-330. 